\title{
GCU
}

Glasgow Caledonian

University

University for the Common Good

\section{Two approaches for the modified asymmetrically clipped optical orthogonal frequency division multiplexing system}

Mohamed, Salma D.; S. Khallaf, Haitham; Shalaby, Hossam; Andonovic, Ivan; Aly, Moustafa H.

Published in:

2013 Second International Japan-Egypt Conference on Electronics, Communications and Computers (JECECC)

DOI:

10.1109/JEC-ECC.2013.6766400

Publication date:

2014

Document Version

Author accepted manuscript

Link to publication in ResearchOnline

Citation for published version (Harvard):

Mohamed, SD, S. Khallaf, H, Shalaby, H, Andonovic, I \& Aly, MH 2014, Two approaches for the modified asymmetrically clipped optical orthogonal frequency division multiplexing system. in 2013 Second International Japan-Egypt Conference on Electronics, Communications and Computers (JEC-ECC). IEEE, pp. 135-139, Second International Japan-Egypt Conference on Electronics, Communications and Computers, Cairo, Egypt, 17/12/13. https://doi.org/10.1109/JEC-ECC.2013.6766400

\section{General rights}

Copyright and moral rights for the publications made accessible in the public portal are retained by the authors and/or other copyright owners and it is a condition of accessing publications that users recognise and abide by the legal requirements associated with these rights.

Take down policy

If you believe that this document breaches copyright please view our takedown policy at https://edshare.gcu.ac.uk/id/eprint/5179 for details of how to contact us. 


\title{
Two Approaches for the Modified Asymmetrically Clipped Optical Orthogonal Frequency Division Multiplexing System
}

\author{
Salma D. Mohamed ${ }^{(1)}$, Haitham S. Khallaf $^{(2)}$, $\operatorname{Hossam~Shalaby~}^{(2)}$, Ivan Andonovic ${ }^{(1)}$, Moustafa H. Aly ${ }^{(3)}$ \\ (1) Electronics and Electrical Engineering Department, Strathclyde University, Glasgow, Scotland, Salma.mohamed@strath.ac.uk \\ (2) Department of Electronics and Communications Engineering, Egypt-Japan University of Science and Technology (E-JUST), \\ Alexandria, Egypt. \\ (3) Electronics and Communications Department, College of Engineering, Arab Academy for Science, Technology, and Maritime \\ Transport, Alexandria, Egypt.
}

\begin{abstract}
Two approaches for Modifying the performance of asymmetrically-clipped optical orthogonal frequency-division multiplexing (ACO-OFDM) technique are proposed. These two approaches involve the use of unipolar encoding which facilitates transmission through optical channels. Monte Carlo simulations have been carried out for the proposed system to evaluate its bit error rate (BER) performance. Our simulation results are then compared to that of both conventional ACO-OFDM and bipolar OFDM systems. It turned out that the proposed modifications improve the BER performance when compared to that of traditional systems. Specifically, in case of the first approach the performance of ACO-OFDM scheme can be improved by more than $3 \mathrm{~dB}$ when adopting the proposed modifications. In addition these modifications would eliminate the reduction in the BER performance between ACO-OFDM and bipolar OFDM and surpass its performance.
\end{abstract}

Keywords- Asymmetrically clipped optical orthogonal frequency division multiplexing (ACO-OFDM), DC-biased optical OFDM (DCO-OFDM), unipolar OFDM (U-OFDM), multi-level quadrature amplitude modulation (M-QAM).

\section{Introduction}

The concept of orthogonal frequency division multiplexing (OFDM) has been existing in research papers for several decades, but only recently it has moved into practice in modern communications systems [1]. Because of its many advantages, OFDM has been successfully adopted in many wired and wireless communications systems [1]-[4]. Indeed OFDM is spectrally efficient, has an inherent robustness against narrowband interference, has a simple equalization technique compared to single-carrier systems, and has excellent robustness in multi-path environments. In addition, by adding a cyclic prefix to the beginning of the OFDM symbol, it can mitigate both the intersymbol and intercarrier interferences as cyclic prefix preserves orthogonality between sub-carriers [1]-[4].

In spite of the widespread use of OFDM in wireless communications and its advantages, it has only recently been used in optical communications [5]. This is because the development in digital signal processing (DSP) technology makes it possible combat the dispersion in optical media, which would allow the transmission at very high data rates through these dispersive media [5]. There are two types of optical OFDM systems, coherent optical OFDM (CO-OFDM) and direct detection non-coherent optical OFDM. In case of coherent optical OFDM, a laser source is used at the receiver to locally generate the carrier, but it is sensitive to phase noise. While in case of direct detection optical OFDM (DDOOFDM) the optical carrier is transmitted with the OFDM signal, and has much simpler receiver but it requires more optical power and the use of guard band between the optical carrier and the OFDM subcarriers. In this paper we are interested in the second type DD-OOFDM [5]-[6].

Since intensity modulation and direct detection is used in noncoherent optical systems and due to the fact that OFDM signal is complex, several techniques have been used to convert this OFDM signal into a real and positive signal that can be used to modulate the optical source and then transmitted through the optical channel. Hermitian symmetry is employed to ensure that the OFDM signal is real but the signal is still bipolar [7]-[16]. In order to make the signal totally positive, many techniques have been proposed in literature, e.g., DCbiased optical OFDM (DCO-OFDM) [7]-[10], asymmetrically-clipped optical OFDM (ACO-OFDM) [7][16], and unipolar OFDM (U-OFDM) [17]. In all these techniques, Hermitian symmetry is employed such that the output of the IFFT block is real. The DCO-OFDM adds DC bias to the bipolar OFDM signal to decrease the negativity of the signal, if there is still negative parts; they are clipped and set to zero. This introduces clipping distortion which according to Bussgang's theorem [7]-[8] and the central limit theorem (CLT) [6]-[11] can be modeled as an attenuation of the data carrying subcarriers and addition of Gaussian noise [6]-[ 8]. The net result is an increase of the optical power, which in some applications may be a constraint [7]-[8].

ACO-OFDM has proven to be more power efficient than DCO-OFDM because it does not require a DC bias so the needed power is much less than DCO-OFDM [14]. In the case of ACO-OFDM, data is mapped only to the odd subcarriers and even subcarriers are set to zero. In addition, the negative parts of the IFFT output are clipped to zero. The clipping noise falls on the even subcarriers and does not affect the odd subcarriers. On the other hand, transmitting data on 


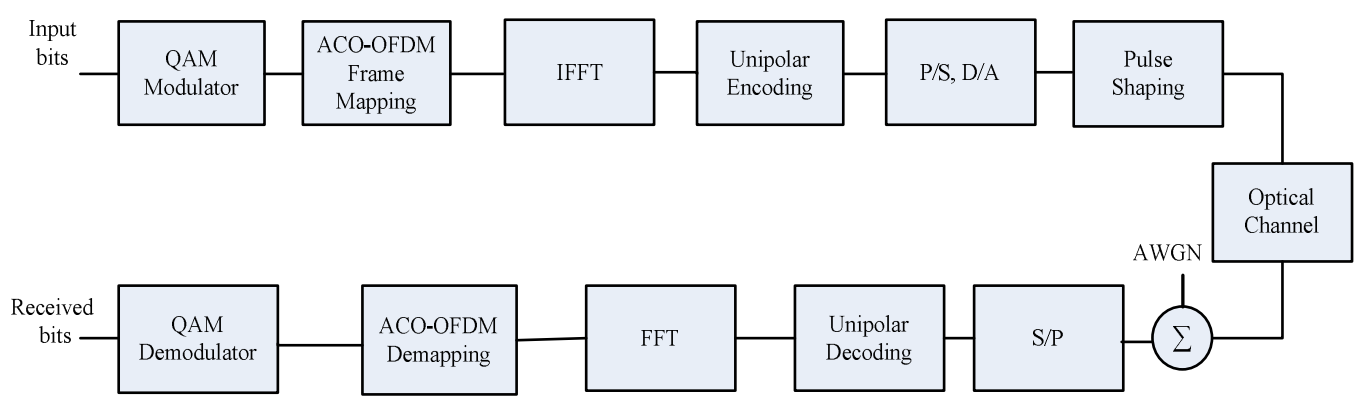

Fig. 1. Modified ACO-OFDM block diagram.

the odd subcarriers would decrease the data rate to half and hence would have half the spectral efficiency of the DCOOFDM for the same order M-QAM. However, it has shown to be more power efficient than DCO-OFDM technique as it does not include a DC- bias [7]-[16]. Furthermore, it has a 3 $\mathrm{dB}$ difference between it and bipolar OFDM, which can be eliminated in our proposed system. Another new technique that has been proposed recently is Unipolar OFDM (UOFDM) suggested by Dobroslav, Sinan, and Harlad which resembles Flip OFDM [17]-[18]. The encoding used by Dobroslav was employed with DC-clipped optical OFDM (DCO-OFDM). This technique has been adopted in an attempt to close the gap between ACO-OFDM and OFDM for bipolar signals, through the generation of unipolar signals without the need to add a DC bias. It starts with the modulation of the conventional OFDM signal until a real bipolar signal is obtained from the IFFT block, then unipolar encoding takes place as explained later in the proposed system. Our proposed modification to conventional ACO-OFDM system improves the BER performance by $3 \mathrm{~dB}$.

The rest of the paper is organized as follows. In Section I, we introduce our proposed system model. In Section II, we introduce our first approach. Second approach is introduced in section III. Section IV is devoted to the simulations of the system performance (in terms of bit-error rate) and discussions of results for the two approaches. Finally, the conclusion is presented in Section V.

\section{SYSTEM MODEL}

Figure 1 shows the block diagram of the proposed system. In standard OFDM systems, the input bits at the transmitter are first mapped to complex M-QAM symbols $A(l)$. These symbols are then fed to the IFFT block, the output being complex and bipolar; but as the signal is intensity modulated the time domain signal has to be real and positive. ACOOFDM technique is employed [9]-[11], [16] to achieve this. For IFFT/FFT of size $N$, the OFDM frame has $N$ subcarriers, so the OFDM signal is defined by $A_{\text {frame }}(m), m=0,1,2,3, \ldots . ., N-1 . \quad$ In the case of ACOOFDM, data is only mapped on the odd subcarriers and the even ones are set to zero such that only $N / 4$ symbols of $A(l), l=0,1,2,3, \ldots . N / 4-1$ are mapped onto half of the odd subcarriers, $\quad A_{\text {frame }}(m), m=1,3,5, \ldots . ., N / 2-1$. Hermitian symmetry [7]-[16] is then imposed on the other half of the subcarriers to ensure that the output of the IFFT is real. The ACO-OFDM vector has the following form:

$$
\begin{aligned}
& A_{\text {frame }}(m)=\left[0 A_{1} 0 A_{3} 0 \ldots \ldots . A_{N / 2-3} 0 A_{N / 2-1} 0\right. \\
& \left.A_{N / 2-1}^{*} 0 A_{N / 2-3}^{*} 0 \ldots \ldots . .0 A_{3}^{*} 0 A_{1}^{*}\right]
\end{aligned}
$$

such that

$$
A_{m}=A_{N-m}^{*}
$$

and

$$
A_{0}=A_{N / 2}=0
$$

After the Hermitian symmetry is employed, the ACO-OFDM signal is passed through the IFFT block. The output time domain signal of the IFFT, $x(n)$ is real but bipolar. This time domain signal is anti-symmetric around the element $N / 2$ as can be seen in Fig. 2 [16].

$$
x(n)=\frac{1}{\sqrt{N}} \sum_{m=0}^{N-1} A_{\text {frame }}(m) \exp \left(\frac{j 2 \pi m n}{N}\right)
$$

such that

$$
x(n)=-x(n+N / 2)
$$

In conventional ACO-OFDM, the bipolar real time domain signal $x(n)$ is clipped at zero level to ensure that the signal is 


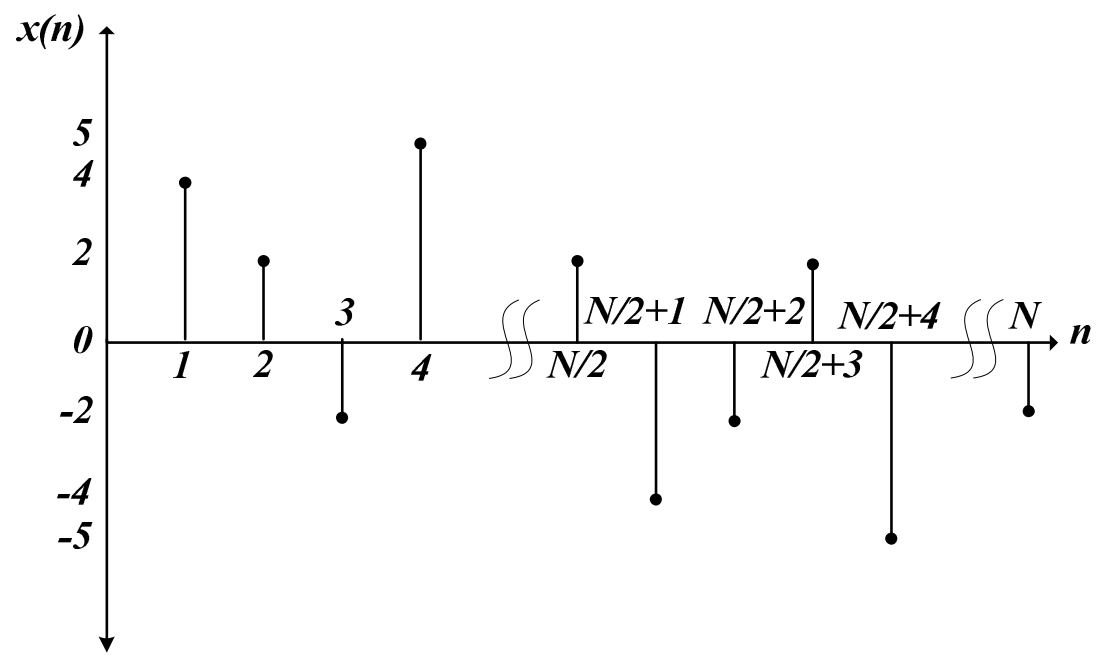

Fig. 2. Real OFDM time domain signal.

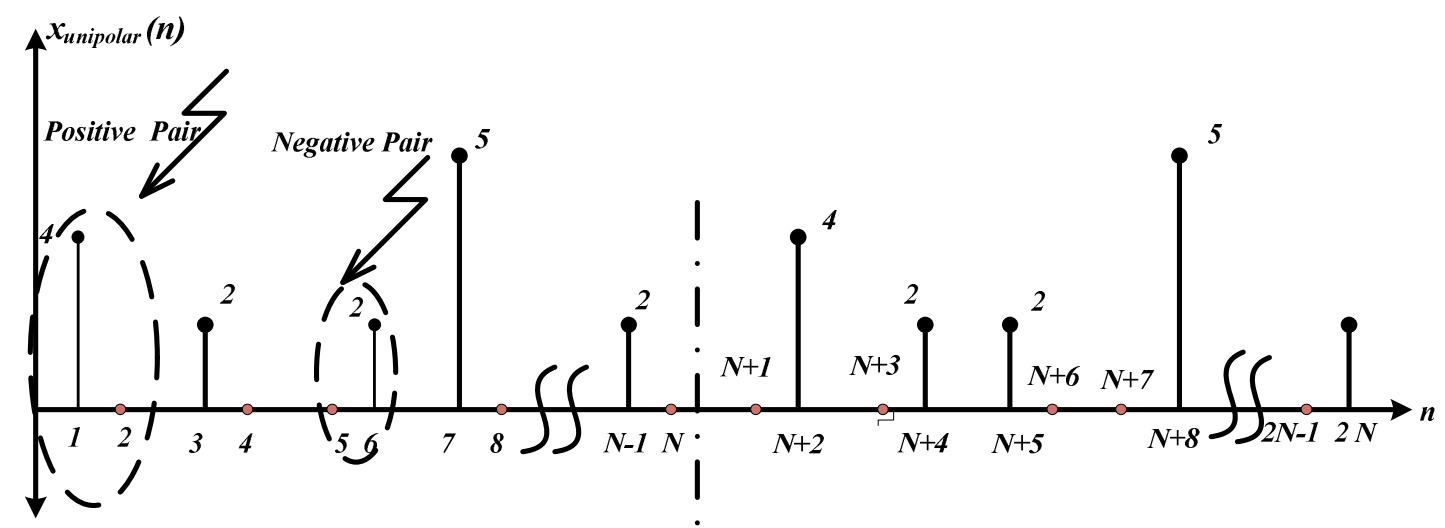

Fig.3 Modified ACO-OFDM signal.

positive. In the proposed system, the unipolar encoding technique is used instead of clipping the signal at zero level. The unipolar encoding represents each sample of the bipolar time domain signal by a pair of new time samples. If the time sample is positive then the first sample of the new pair is set with its amplitude, and the second sample of the new pair is set to zero, and if the original time domain sample is negative then the first sample of the new pair is set to zero and the second sample of the new pair is set with its positive amplitude, Fig. 3. There are two approaches for these modifications.

\section{FIRST APPROACH}

The output of the unipolar encoder xunipolar(n) is then fed to the pulse shaping block, then the optical source is intensity modulated by the output of the pulse shaping block xmodified_ACO $(\mathrm{t})$.

$x_{\text {modified_ACO }}(t)=\sum_{m=0}^{\infty} \sum_{j=0}^{2 N-1}\left(x_{\text {unipolar }}\right)_{j}^{m} g(t-n T-2 m N T)$ where $\mathrm{m}$ is the OFDM frame number, $\mathrm{T}$ is the channel symbol time, and $g(t)$ is the pulse shaping function. $g(t)$ is assumed to be rectangular pulse of unity amplitude and a duration of $\mathrm{T}$ and centered at $\mathrm{t}=0$.

$$
g(t)=\prod(t / T)=\left\{\begin{array}{l}
1,-T / 2 \leq t \leq T / 2 \\
0, \text { otherwise }
\end{array}\right.
$$

By this modification, the length of the frame will be doubled so the spectral efficiency will be half that of the conventional ACO-OFDM due to the unipolar encoding [17].

In our simulation, it is assumed that the optical source is ideal, such that a linear transformation between the input and output optical power is achieved. The optical signal transmitted through the optical channel is subject to AWGN [5]-[16]. Of course at the receiver side, the signal becomes bipolar due to the fact that the noise is added in the electrical domain [5]. This model is usually used in the wireless optical systems where the obvious degradation in the signal is owing to the high level of ambient infrared radiation producing shot noise at the receiver, which can be modeled as AWGN. The received signal $\mathrm{y}(\mathrm{t})$ can expressed as [16]:

$$
y(t)=h(t) * x_{\text {modified_ACO }}(t)+v(t)
$$


where $*$ denotes the convolution operation, $\mathrm{h}(\mathrm{t})$ represents the impulse response of the optical channel, and $v(t)$ is the AWGN. In this paper the performance of conventional ACOOFDM and bipolar OFDM is compared to that of the proposed system subject to AWGN, so without loss of generality $y(t)$ takes the form [16]:

$$
y(t)=x_{\text {modified_ACO }}(t)+v(t)
$$

Sampling at $t n m=n T+m N T$, we get the samples fed to the unipolar decoder. As each pair denotes the sign and amplitude of the original signal, unipolar decoder detects which sample of each pair is active to determine the sign of the original signal, neglecting the passive sample. This can be achieved by comparing the amplitudes of samples in each pair; the higher amplitude sample considered active and the other passive [17]. Then using the fact that the OFDM signal is anti-symmetric about the N/2 th element, by detecting which element is bigger than the other and then averaging takes place

$$
\begin{aligned}
& x \quad(m)= \begin{cases}\frac{x(m)-x(m+N / 2)}{2} & \text { if } x(m)>x(m+N / 2) \\
-\frac{(-x(m)+x(m+N / 2))}{2} & \text { otherwise }\end{cases} \\
& x \quad(m+N / 2)= \begin{cases}-\frac{(x(m)-x(m+N / 2))}{2} & \text { if } x(m)>x(m+N / 2) \\
\frac{(-x(m)+x(m+N / 2))}{2} & \text { otherwise }\end{cases}
\end{aligned}
$$

\section{SECOND APPROACH}

As the output of IFFT $x(n)$ is anti-symmetrical around the $\mathrm{N} / 2$ th element, so instead of repeating the elements again with opposite sign, we will sacrifice half of the OFDM elements in each frame after the N/2th element. After that the signal will be encoded and then pass by the pulse shaping block. In this case, the length of the modified ACO-OFDM frame will be the same as that of conventional ACO-OFDM. So the spectral efficiency of both of them will be the same.

\section{Simulation RESUlts}

Monte Carlo simulations are conducted for 4, 16, 64, 256QAM to calculate the average bit error rate (BER) performance. All simulations are conducted for 1024 point IFFT/FFT modified ACO-OFDM system and repeated for 10000 iterations and then average BER are calculated. As no fading is assumed in IM/DD systems, the evaluation of the performance of modified ACO-OFDM subject to AWGN can be considered a sufficient model that treats optical wireless systems. As will be described below our proposed modifications improve the BER performance of ACO-OFDM.

\section{A. First Approach}

Indeed we show that its performance is better than that of bipolar OFDM due to the unipolar detection executed in time domain. In fact the proposed modifications eliminate the 3-dB difference that was found between conventional ACO-OFDM and bipolar OFDM. Figure 4 compares the BER performance of conventional ACO-OFDM and the proposed modified ACO-OFDM. In summary in the case of 4-QAM, the performance of the modified system compared with conventional ACO-OFDM is better by $\sim 3 \mathrm{~dB}$. In the case of 16-QAM, the BER of the modified ACO-OFDM is better by 4 $\mathrm{dB}$ compared to that of conventional ACO-OFDM. The improvement increases as the constellation increases. Fig. 5 compares the performance of modified ACO-OFDM to that of traditional bipolar OFDM. The performance not only eliminates the 3-dB difference between conventional ACOOFDM and bipolar but it also outperforms bipolar OFDM.

\section{B. Second Approach}

In case of the second approach, the modification in performance will be much less than in the first approach as the averaging step will be cancelled, but there will be still a modification. Figure 6 is the comparison between the BER performance of the second approach and conventional ACOOFDM. The performance of the former is better than the latter, and the improvement increases as the constellation size increases due to the encoding.

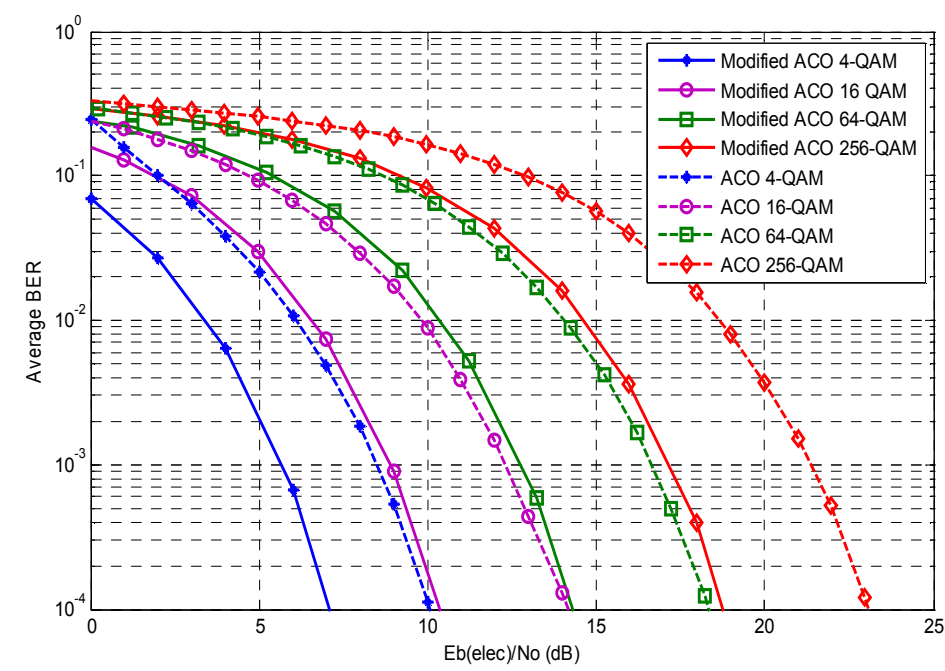

Fig. 4. Bit-error rate comparison between proposed Mod. ACO-OFDM and conventioanl ACO-OFDM.

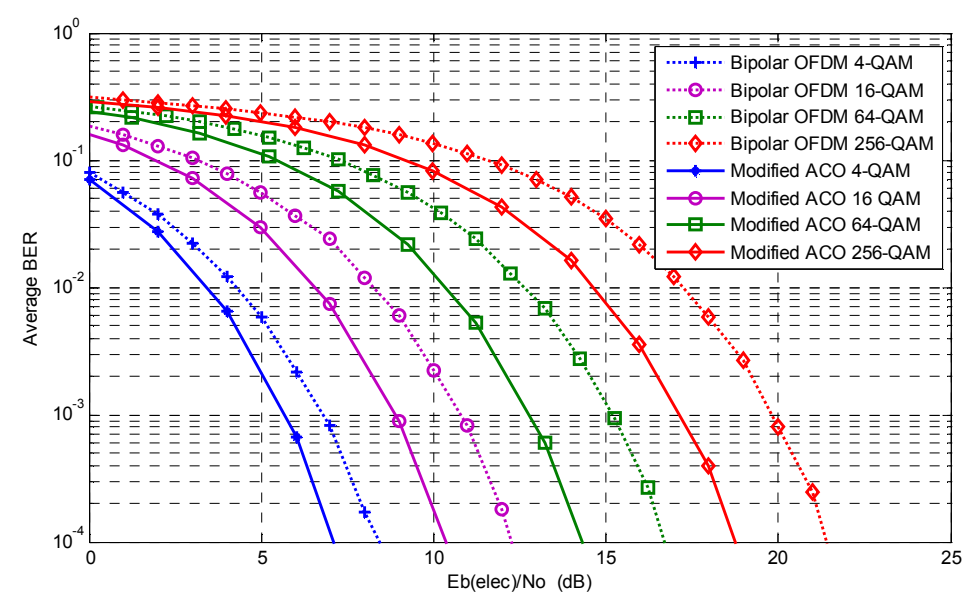

Fig. 5 Bit-error rate comparison between proposed modified ACO-OFDM and bipolar. 


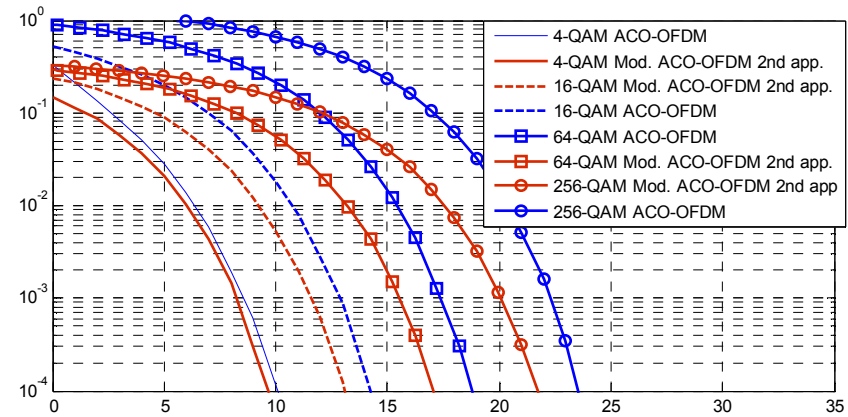

Fig.6 Bit-error rate comparison between proposed Mod. ACOOFDM 2nd approach and conventional ACO-OFDM.

\section{CONCLUSION}

Two approaches for a modification to conventional ACOOFDM scheme using unipolar encoding have been proposed. The BER performance of the first approach is simulated and compared to that of both conventional ACO-OFDM and bipolar OFDM techniques. Our results reveal that the performance of ACO-OFDM scheme can be improved by more than $3 \mathrm{~dB}$ when adopting the proposed modifications. In case of the second approach, there is an improvement in the performance that reaches $2 \mathrm{~dB}$ in case of 256-QAM.

\section{REFERENCES}

[1] Richard Van Nee and Ramjee Prasad," OFDM For Wireless Multimedia Communications," Artech House, 2000

[2] Henrick Schuzle, and Christian Luders, " Theory and Applications of OFDM and OCMA Wideband Wireless Communications," John Wiley, 2005 .

[3] Savo Glisic," Advanced WirelessCommunications 4G Technologies," John Wiley, 2004.

[4] Upamanyu Madhow," Fundamentals of Digital Communications," Cambridge University Press, 2008.

[5] Jean Armstrong," OFDM for Optical Communications," J. Lightw. Technol., vol. 27, no. 3, pp. 189-204, Feb. 2009.

[6] William Shieh, Ivan Djordjevic," OFDM for Optical Communications," Elsevier press, 2010

[7] Liang Chen, Brian Krongold, and Jamie Evans, "Performance Evaluation of Optical OFDM Systems with Nonlinear Clipping Distortion",IEEE ICC09, June, 2009.

[8] Svilen Dimitrov, Sinan Sinanovic, and Harald Haas," A Comparison of OFDM- based Modulation Schemes for OWC with Clipping Distortion," 2nd IEEE Workshop, 2011.

[9] Svilen Dimitrov, Sinan Sinanovic, and Harald Haas," Clipping Noise in OFDM- Based Optical Wireless Communication Systems," IEEE Trans. On Commun., vol. 60, no. 4, pp. 1072-1081, April 2012.

[10] Jean Armstrong, and Brendon J. C. Schmidt,"Comparison of Asymmetrically Clipped Optical OFDM and DC-Biased Optical OFDM in AWGN", IEEE Commun. Letters, Vol. 12, no. 5, pp. 343-345, May 2008.

[11] Liang Chen, Brian Krongold, and Jamie Evans," Diversity Combining for Asymmetrically Clipped Optical OFDM in IM/DD Channels," IEEE GLOBECOM, pp. 1-6, Dec. 2009.

[12] Sarangi D. Dissanayake, Kusha Panta, and Jean Armstrong, "A Novel Technique to Simultaneously Transmit ACO-OFDM and DCO-OFDM in IM/DD Systems," GLOBECOM Workshop on Optical Wireless Commun, pp. 782 - 786, Dec. 2011.

[13] J. Armstrong , and A.J. Lowery,"Power Efficient Optical OFDM," Electronics Letters, Vol. 42, no. 6, pp. 370-372, March 2006.

[14] Sarah Kate Wilson, and Jean Armstrong," Transmitter and Receiver Methods for Improving Asymmetrically- Clipped Optical OFDM,' IEEE Trans. Commun., vol. 8,no. 9, pp. 4561 - 4567 Sep. 2009.

[15] Svilen Dimitrov, Sinan Sinanovic, and Harald Haas, "Double-sided Signal Clipping in ACO-OFDM Wireless Communication Systems,' IEEE ICC, pp. 1-5, June 2011

[16] Jean Armstrong, Brendon J.C. Schmidt, Dhruv Karla, Himal A. Suraweera, and Arthur J. Lowery," Performance of Asymmetrically Clipped Optical OFDM in AWGN for an Intensity Modulated Direct Detection System," GLOBECOM'06 IEEE, pp. 1-5, Nov. 2006.

[17] Dobroslav Tsonev, Sinan Sinanovic, and Harald Haas," Novel Unipolar Orthogonal Frequency Division Multiplexing (U-OFDM) for Optical Wireless," VTC spring, IEEE 75 ${ }^{\text {th }}$, pp. 1-5, May 2012.

[18] Nirmal Fernando, Yi Hong, and Emanuele Viterbo," Flip-OFDM for Optical Wireless Communications ," IEEE ITW, pp. 5-9, Oct., 2011. 